\title{
Changes in immune activation in the T Cell compartments of HIV HCV coinfected patients during PEG IFN RBV treatment
}

\author{
Amélie Menard*, Corinne Brunet, Véronique Obry, Patrick Dukan, Sylvie Brégigeon, Olivia Faucher, Estelle Balducci, \\ Anne-Suzel Ritleng, Françoise Dignat-George, Isabelle Poizot Martin
}

From 17th International Symposium on HIV and Emerging Infectious Diseases (ISHEID) Marseille, France. 23-25 May 2012

Chronic activation of CD8 T-cell compartment is critical during HCV-HIV-1 coinfection. The objective of this study was to evaluate the impact of pegylated-interferon (PEG-IFN) in combination with ribavirin (RBV) on immune activation in HCV-HIV-coinfected patients.

T cell phenotype (CD8+CD38+,CD8+DR+) was quantified using flow cytometry analysis,. Measurements were performed at day one of treatment (Baseline, BL), then at week (W)12,W24,W48 and W24 post-treatment. HVC viral load was measured using a PCR (COBAS TaqMan 48; Roche), exhibiting a limit of detection at12 $\mathrm{IU} / \mathrm{ml}$. Statistical analysis was performed with SPSS 17.0.

11 pts (64\% of males; median age 47.4 [45.1-51]) with a median follow up for HCV infection of 14.7y [11.7; 19.1]) were evaluated. All were treated for HIV infection (PI- based regimen: 63.6\%) with an HIV-VL < 40copies/ ml. Median CD4 and CD8 T cells count at BL was 886/ $\mathrm{mm} 3$ [671; 1008] and $825 / \mathrm{mm} 3$ [530; 1843], respectively. HCV genotype was 1 for $63.6 \%, 3$ in $27.3 \%$ and 4 in one pt. HCV VL at BL was 5.9 [4.6; 6.7] (log UI/ml). Up to now, 2 pts stopped HCV treatment at W2 and W4, 9 pts have reached W24, and 5 of them are between W24 and W48. The results at W12 and W24 are presented in the table. We observed a significant decrease of the number of circulating total lymphocytes and CD4T cells in absolute value $(\mathrm{p}=0.008)$, but a significant increase in the percentage of CD4+ T-cells and a significant decrease in the percentage of CD8 $+\mathrm{T}$ cells at W24. HCV VL was negative for all of them.

These preliminary results show that the immune system hyperactivation driving by HCV disease can be reduced with a control of HCV replication. However, we observed a discrepancy in the evolution of CD8+CD38+ and CD8 $+\mathrm{DR}+$ expression at W12 which remains at W24. These results have to be confirmed with the next measurement performed at W48 and W24 post treatment.

Published: 25 May 2012

doi:10.1186/1742-4690-9-S1-P56

Cite this article as: Menard et al:: Changes in immune activation in the

T Cell compartments of HIV HCV coinfected patients during PEG IFN

RBV treatment. Retrovirology 2012 9(Suppl 1):P56.

Table 1

\begin{tabular}{llllll}
\hline & BL & W12 & p & W24 & p \\
\hline CD4+ T Cell (\%) & $27.0[24.5 ; 37.1]$ & $37.5[26.8 ; 46.0]$ & 0.008 & $43.0[30.5 ; 47.3]$ & $\mathbf{0 . 0 1 1}$ \\
CD8+ T Cell (\%) & $46.0[30.4 ; 51.0]$ & $43.2[25.9 ; 49.7]$ & 0.110 & $37.4[26.5 ; 46.9]$ & $\mathbf{0 . 0 2 8}$ \\
DR+CD8+ T Cell (\%) & $11.0[6.4 ; 19.5]$ & $5.2[2.8 ; 9.9]$ & 0.017 & $2.4[1.4 ; 7.4]$ & $\mathbf{0 . 0 0 8}$ \\
CD38+CD8+ T Cell (\%) & $14.7[6.8 ; 21.5]$ & $43.2[28.9 ; 48.9]$ & 0.008 & $36.2[15.7 ; 47.1]$ & $\mathbf{0 . 0 1 5}$ \\
\hline
\end{tabular}

Haematology-Aids Unit Hôpital Sainte-Marguerite, Marseille, France

@ 2012 Menard et al; licensee BioMed Central Ltd. This is an Open Access article distributed under the terms of the Creative Commons 